\title{
A Novel Multitemporal InSAR Model for Joint Estimation of Deformation Rates and Orbital Errors
}

\author{
Lei Zhang, Member, IEEE, Xiaoli Ding, Zhong Lu, Senior Member, IEEE, \\ Hyung-Sup Jung, Member, IEEE, Jun Hu, and Guangcai Feng
}

\begin{abstract}
Orbital errors, characterized typically as longwavelength artifacts, commonly exist in interferometric synthetic aperture radar (InSAR) imagery as a result of inaccurate determination of the sensor state vector. Orbital errors degrade the precision of multitemporal InSAR products (i.e., ground deformation). Although research on orbital error reduction has been ongoing for nearly two decades and several algorithms for reducing the effect of the errors are already in existence, the errors cannot always be corrected efficiently and reliably. We propose a novel model that is able to jointly estimate deformation rates and orbital errors based on the different spatial-temporal characteristics of the two types of signals. The proposed model is able to isolate a long-wavelength ground motion signal from the orbital error even when the two types of signals exhibit similar spatial patterns. The proposed algorithm is efficient and requires no ground control points. In addition, the method is built upon wrapped phases of interferograms, eliminating the need of phase unwrapping. The performance of the proposed model is validated using both simulated and real data sets. The demo codes of the proposed model are also provided for reference.
\end{abstract}

Index Terms-Interferometric synthetic aperture radar (SAR) (InSAR), least squares, orbital error, SAR, sparse matrix.

\section{INTRODUCTION}

$\mathbf{M}$ ULTITEMPORAL interferometric synthetic aperture radar (SAR) (InSAR) (MTInSAR) techniques, e.g., persistent scatterer interferometry (PSI) (e.g., [1]-[4]), satellitebased augmentation system [Small BAseline Subset (SBAS)] [5], [6], Coherent Pixels Technique [7], and temporarily coherent point InSAR [8], [9], [32], have been widely applied to derive ground motions with relatively high accuracy (e.g.,

Manuscript received February 22, 2012; revised September 9, 2012, March 15, 2013, and June 22, 2013; accepted June 26, 2013. This work was supported in part by the Research Grants Council of the Hong Kong Special Administrative Region under Project PolyU5154/10E, by the Faculty of Construction and Land Use under the Sustainable Urbanization Research Fund, and by the U.S. Geological Survey Volcano Hazards Programs.

L. Zhang and X. Ding are with The Hong Kong Polytechnic University, Kowloon, Hong Kong (e-mail: 1slzhang@polyu.edu.hk; lsxlding@ polyu.edu.hk).

Z. Lu is with the Cascades Volcano Observatory, U.S. Geological Survey, Vancouver, WA 98683-9589 USA (e-mail: lu@usgs.gov).

H.-S. Jung is with The University of Seoul, Seoul 130-743, Korea (e-mail: hsjung@uos.ac.kr).

J. Hu is with Central South University, Changsha 410083, China, and also with the Department of Land Surveying and Geo-Informatics, The Hong Kong Polytechnic University, Kowloon, Hong Kong (e-mail: csuhujun@csu.edu.cn).

G. Feng was with Central South University, Changsha 410083, China. $\mathrm{He}$ is now with the Crustal Deformation and InSAR Group, King Abdullah University of Science and Technology, Thuwal 23955-6900, Saudi Arabia (e-mail: fredgps@gmail.com).

Color versions of one or more of the figures in this paper are available online at http://ieeexplore.ieee.org.

Digital Object Identifier 10.1109/TGRS.2013.2273374 subcentimeters) and under all-weather conditions. The MTInSAR techniques use SAR data acquired over the same area on different dates to suppress the effects of atmospheric delays, terrain height errors, and potential phase-unwrapping errors [10]. Orbital error reduction is a required step in the utilization of InSAR measurements [11], and the need for orbit refinement becomes even more pressing when dealing with SAR data acquired by the Japanese Earth Resources Satellite 1 [12], Radarsat-1/2[13], [14], and Advanced Land Observing Satellite (ALOS)/Phased Array type L-band Synthetic Aperture Radar (PALSAR) [15]. In fact, without accurate estimate of orbital errors, there is no basis for precise retrieval of deformation signals from InSAR phases. However, it appears that the issue of orbital errors has not yet been given sufficient attention in the current MTInSAR techniques. For example, there is no telling consideration in the PSI system on the refinement of orbital errors except for taking the phase component due to orbit indetermination as a part of atmospheric contribution [1]. In the SBAS method, the orbit errors are fitted to a plane and removed from the unwrapped phase of each interferogram. The best fit plane may or may not fully reflect the effect of the orbit errors. More recently, an orbit correction method based on the analysis of phase gradients in the wrapped interferograms has been integrated into the SBAS processing chain [16]. It is, however, not able to effectively separate the long-wavelength deformation signal from the orbit errors.

Understanding the characteristics of orbital errors is the key to determining how the errors can be effectively modeled. In the spatial domain, the phase component due to orbit errors usually appears in an interferogram as a smoothly varying pattern. The long-wavelength orbital artifacts are obvious in some cases but can be obscured by other phase components in the other cases. When the orbit errors are visible in an interferogram, a 2-D polynomial is commonly used to model and remove the errors [17], [18]. However, the signals of atmospheric delay and ground deformation may affect the determination of the coefficients of the polynomial. Fig. 1 shows the estimated orbital phase polynomial from a simulated interferogram that also contains atmospheric artifacts [Fig. 1(c)] and a deformation signal [Fig. 1(e)]. The long-wavelength deformation signal that mimics the effect of the orbit errors can seriously distort the estimated orbit errors. This suggests that the orbit errors can be particularly troublesome in mapping long-wavelength ground deformation such as interseismic motions across active faults. On the other hand, the orbit errors in MTInSAR images are associated with particular time epochs; they have rather weak temporal correlation compared with the deformation signal [19], [20]. 
(a)

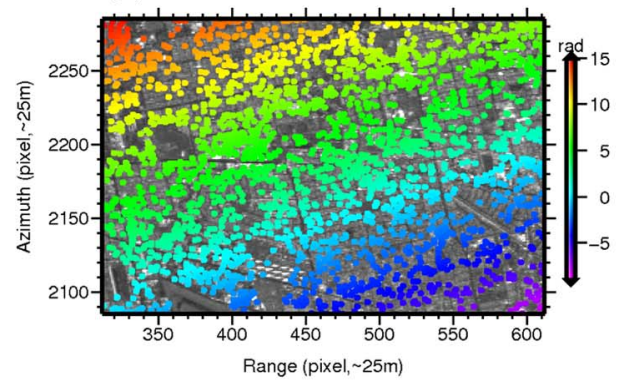

(d)

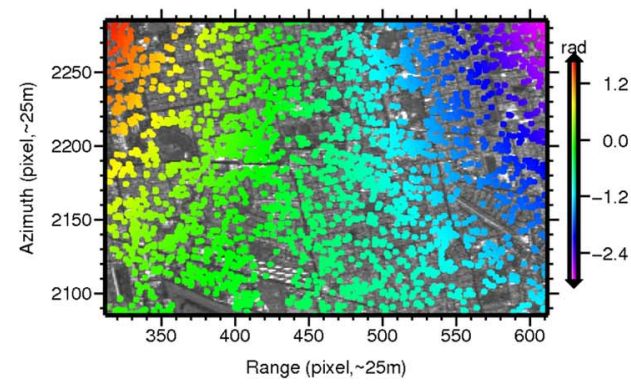

(b)

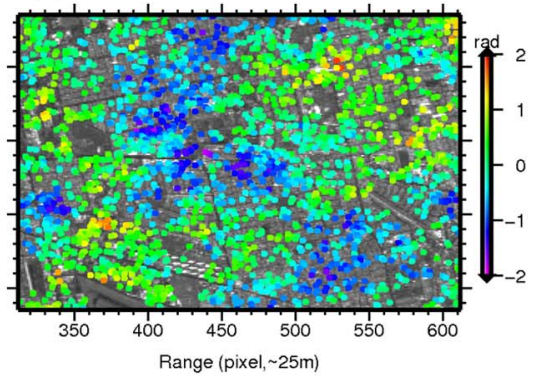

(e)

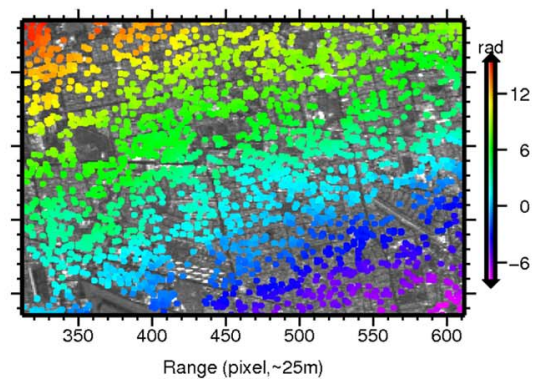

(c)

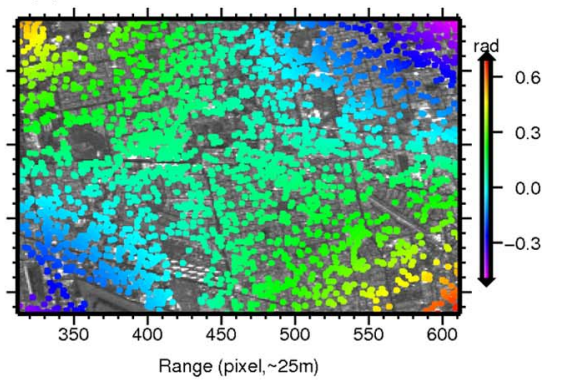

(f)

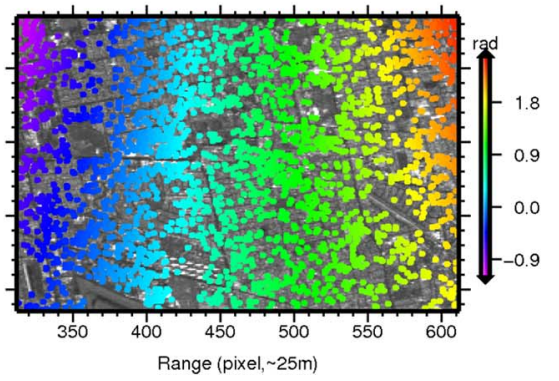

Fig. 1. Effects of atmospheric delay and long-wavelength deformation signals on the estimation of the polynomial for the orbital error. (a) Simulated orbital error in SAR pixel coordinates. (b) Simulated atmospheric artifacts. (c) Effect of polynomial fitting caused by atmospheric artifacts. The orbital error in (a) and the atmospheric delay in (b) are combined. The result is fitted with a polynomial, and its difference with the polynomial fitting using the orbit error alone is shown in (c). (d) Simulated deformation phase. (e) Combination of (a) orbital error, (b) atmospheric artifacts, and (d) deformation. (f) Similar to (c), the polynomial fitting residual on (e).

Based on the aforementioned characteristics of the orbit errors, it is more advantageous to make use of a stack of MTInSAR images to model and remove the errors. Instead of focusing only on a single image pair, Biggs et al. [20] proposed a network method to separate the orbit errors from a set of unwrapped phases. Since unwrapped phases are used as the input in the proposed model, the potential phase-unwrapping errors may distort the estimated orbit errors. In addition, the method was initially designed for analyzing interseismic strain accumulation, and it requires iterations and a geophysical model. However, for large ground motions with complex spatial-temporal patterns, the iterative procedure is of low efficiency and may diverge during parameter retrieval. Since the orbital phase errors in an interferogram are equivalent to phase contribution due to a baseline error, Bähr and Hanssen [21] proposed another network approach that transfers the estimation of orbit errors to the estimation of baseline errors. However, the approach works under the condition that the orbit errors are the only remaining signal in the interferograms and cannot effectively separate orbit errors from large-scale ground motions, neither the wavelet-based method for orbital error correction [30] can.

The primary goal of this paper is to provide a novel MTInSAR model that can jointly estimate the deformation rates and the orbit errors from a set of multimaster interferograms with short baselines. The model is based on the different spatial-temporal characteristics of the deformation signal and the orbit errors. The deformation component correlates with the time span of an interferogram, while the orbital phase errors depend only on the time of the SAR acquisition and can be spatially modeled by a low-order polynomial. Following the framework proposed in [8], the parameters (i.e., deformation rates, digital elevation model (DEM) errors, and orbital phase) are resolved directly from wrapped phase, and phase unwrapping is not required. Both simulated and real data sets will be used to evaluate the performance of the proposed model.

\section{Methodology}

\section{A. Observations}

We select to use a set of interferograms with short baselines (spatial and temporal baselines and Doppler centroid difference) and take the differential phase at the arcs (coherent point pairs) as the basic observations. The coherent point pairs should be densely constructed according to the strategy proposed in [8]. The advantage of using arcs is that the differencing operation can reduce the spatially correlated atmospheric signals. The remaining atmospheric artifacts at the arcs are taken as random noise in the temporal domain [1].

Considering $M$ differential interferograms generated from $N$ SAR images, the wrapped phase at the coherent point $P$ in the $i$ th interferogram can be written as

$$
\begin{aligned}
\phi_{p}^{i}=W\{ & \phi_{\mathrm{topo}, p}^{i}+\phi_{\mathrm{defo}, p}^{i}+\phi_{\mathrm{atmo}, p}^{i} \\
& \left.+\phi_{\mathrm{orbit}, p}^{i}+\phi_{\text {noise }, p}^{i}\right\}, \quad i=1, \ldots, M
\end{aligned}
$$

where $W\{\cdot\}$ is the wrapping operator, $\phi_{\text {topo, } p}^{i}$ is the phase related to topographic error, $\phi_{\mathrm{defo}, p}^{i}$ is the phase component due to ground motion, $\phi_{\text {atmo, } p}^{i}$ is the phase associated with atmospheric delay, $\phi_{\text {orbit, } p}^{i}$ is the phase caused by orbital error, and $\phi_{\text {noise }, p}^{i}$ is the noise (due mainly to decorrelation effects). For an arc constructed by two neighboring coherent points (for 
example, $p$ and $q$ ), the phase difference between $p$ and $q$ can be expressed as

$$
\begin{aligned}
\Delta \phi_{p, q}^{i}= & W\left\{\Delta \phi_{\mathrm{topo}, p, q}^{i}+\Delta \phi_{\mathrm{defo}, p, q}^{i}+\Delta \phi_{\mathrm{atmo}, p, q}^{i}\right. \\
& \left.+\Delta \phi_{\mathrm{orbit}, p, q}^{i}+\Delta \phi_{\text {noise }, p, q}^{i}\right\}, \quad i=1, \ldots, M .
\end{aligned}
$$

If there are $G$ arcs in total constructed from $P$ coherent points, the observations from the $i$ th interferogram can be written as

$$
\Delta \boldsymbol{\Phi}^{i}=\left[\begin{array}{llll}
\Delta \phi_{1}^{i} & \Delta \phi_{2}^{i} & \cdots & \Delta \phi_{G}^{i}
\end{array}\right]^{\mathrm{T}}, \quad i=1, \ldots, M .
$$

The observations for all interferograms can be denoted by

$$
\underset{(M \times G) \times 1}{\Delta \Phi}=\left[\begin{array}{llll}
\Delta \Phi^{1} & \Delta \Phi^{2} & \cdots & \Delta \Phi^{M}
\end{array}\right]^{\mathrm{T}} .
$$

The vector $\boldsymbol{\Delta} \boldsymbol{\Phi}_{(M \times G) \times 1}$ contains all observations for the proposed model. It is worth noting that, although the observations for the models presented in [1], [2], and [8] are also phase differences at arcs, it is difficult (or impossible) for these models to include the orbital unknowns which are spatially correlated and have the relationship with the location of the coherent points. This is mainly because these models are essentially designed for each single arc and parameters at arcs are first resolved and then integrated with respect to a reference point to obtain the parameters at all coherent points, resulting in no full usage of spatial information. A direct relationship between the observations at arcs and the parameters at coherent points is actually vital for the proposed model, which will be presented in the following sections.

\section{B. Modeling Orbit Errors}

As mentioned previously, orbit errors in an interferogram can be modeled by a low-order polynomial. Since an interferogram is typically a linear combination of two single-look complex (SLC) images, the orbit errors can also be represented by two polynomials, each representing the orbital errors in one of the two SAR images. By estimating a polynomial for each image, the total number of coefficients (unknowns) is usually significantly less than that for estimating a polynomial for each interferogram. In addition, we assume that the orbit errors in one of the images (i.e., the reference image) are negligible. The following polynomial is used to represent the relative orbital error of a pixel $P$ with coordinate $(X, Y)$ with respect to the reference image:

$$
\phi_{\text {orb }, \text { slc }, p}^{j}=a^{j} X+b^{j} Y+c^{j} X Y, \quad j=1, \ldots, N-1
$$

where $a^{j}, b^{j}$, and $c^{j}$ are the unknown coefficients to be estimated. A constant term is not needed since it has the same effect on all pixels in the image and will be canceled out during the differencing operation. The relative orbit errors at all arcs in an SLC image can be written as

$$
\underset{G \times 1}{\Delta \boldsymbol{\Phi}_{\text {slc }}^{j}}=a^{j} \mathbf{d X}+b^{j} \mathbf{d} \mathbf{Y}+c^{j} \mathbf{d X Y}, \quad j=1, \ldots, N-1
$$

where $\mathbf{d X}, \mathbf{d Y}$, and $\mathbf{d X Y}$ are the vectors of pixel coordinate differences between points that form the arcs. Equation (6) can be written in a matrix form

$$
\underset{G \times 1}{\Delta \boldsymbol{\Phi}_{\mathrm{slc}}^{j}}=\underset{G \times 3}{\mathbf{D}^{j}} \mathbf{P}_{\substack{\text { slc,orb } \\ 3 \times 1}}^{j}, \quad j=1, \ldots, N-1
$$

where $\mathbf{D}_{G \times 3}^{j}=\left[\begin{array}{lll}\mathbf{d X} & \mathbf{d} \mathbf{Y} & \mathbf{d} \mathbf{X Y}\end{array}\right]$ and $\mathbf{P}_{\substack{\text { slc,orb } \\ 3 \times 1}}^{j}=\left[\begin{array}{lll}a_{j} & b_{j} & c_{j}\end{array}\right]^{\mathrm{T}}$. It should be noted that a higher order polynomial with more coefficients can also be considered according to the orbit error pattern. Empirically speaking, a full second-order polynomial should be enough for most cases. Let $\mathbf{A}$ be a matrix for differencing operation to generate $\mathbf{M}$ interferograms from $\mathbf{N}$ SLC images, and the column corresponding to the reference image has been removed, which has a form as

$$
\mathbf{A}=\left[\begin{array}{ccccc}
-1 & 0 & 1 & \cdots & 0 \\
0 & -1 & \cdots & 1 & 0 \\
\vdots & \ddots & & & \\
0 & 0 & -1 & \cdots & 1
\end{array}\right]_{M \times(N-1)}
$$

The design matrix relating the observations in (4) and the orbit error parameters in (7) at the arcs is

$$
\underset{(M \times G) \times((N-1) \times 3)}{\mathbf{D}_{\text {orb }}}=\underset{M \times(N-1)}{\mathbf{A}} \otimes \underset{G \times 3}{\mathbf{D}}
$$

where $\otimes$ denotes the Kronecker tensor product. The phase components due to the orbit errors at all arcs of the interferograms can then be obtained

$$
\underset{(M \times G) \times 1}{\Delta \boldsymbol{\Phi}_{\text {orb }}}=\mathbf{D}_{\text {orb }} \underset{((N-1) \times 3) \times 1}{\mathbf{P}_{\text {orb }}}
$$

where $\mathbf{P}_{\text {orb }}$ contains all polynomial coefficients of relative orbital errors with respect to the reference image.

\section{Modeling Deformation Rates and DEM Errors}

Since the relationship between the phase components in interferograms and the deformation rate as well as DEM errors has been well discussed in the literature (e.g., [1]-[10]), we limit ourselves here to a brief description. For a given point $(p)$, in addition to orbit error, the topographic error $\left(\Delta h_{p}\right)$ and the linear deformation rate $\left(v_{p}\right)$ also contribute to the differential phase [8]

$$
\begin{aligned}
& \phi_{\text {topo }, p}^{i}=\alpha_{p}^{i} \Delta h_{p} \\
& \phi_{\text {defo }, p}^{i}=\beta_{p}^{i} v_{p}
\end{aligned}
$$

where superscript $i$ stands for the $i$ th interferogram, and

$$
\begin{aligned}
& \alpha_{p}^{i}=-\frac{4 \pi}{\lambda} \frac{B_{\perp, p}^{i}}{r_{p}^{i} \sin \theta_{p}^{i}} \\
& \beta_{p}^{i}=-\frac{4 \pi}{\lambda}\left(t_{M_{i}}-t_{S_{i}}\right) v_{p} .
\end{aligned}
$$




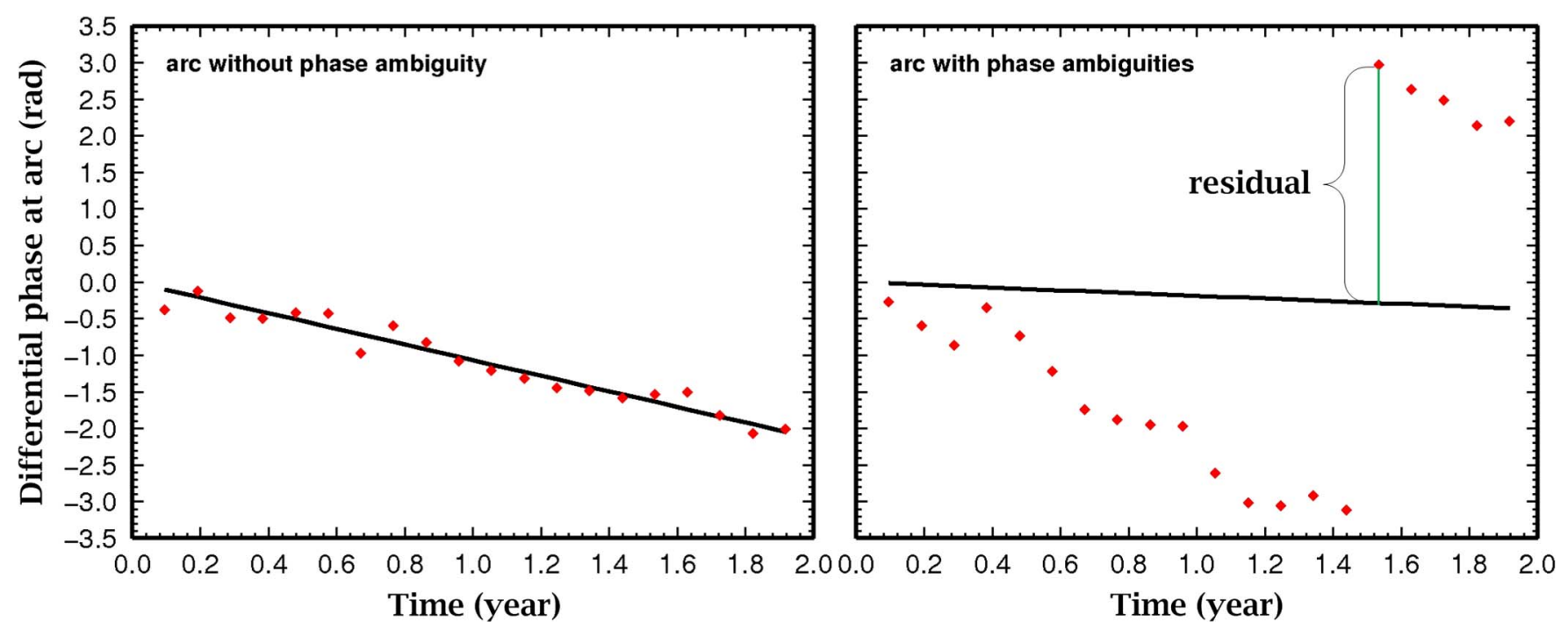

Fig. 2. Illustration of the effect of phase ambiguities on parameter estimation. For visualization purposes, only one parameter is estimated. The red dots are the wrapped differential phases at a given arc. It is clear that phase ambiguities can result in large residuals.

Equation (11) shows the phase components due to the topographic error and the deformation rate, respectively. Readers wishing to delve further into (11) and (12) can refer to [8]. Considering $M$ interferograms in total, the differential phase due to deformation and the topographic error at $p$ can be expressed as

$$
\boldsymbol{\Phi}_{\text {topo }+ \text { defo }, \mathbf{p}}=\underset{M \times 2}{\mathbf{B}}\left[\begin{array}{c}
\Delta h_{p} \\
v_{p}
\end{array}\right]
$$

where $\mathbf{B}_{M \times 2}$ is the design matrix relating the topographic error and the linear deformation rate to the phase observations. Let $\mathbf{C}_{G \times(P-1)}$ be an index matrix indicating the relationship between the $G$ arcs and the $P$ coherent points where the column corresponding to the point with known DEM error and deformation rate (i.e., the reference point) has been removed. The phase differences at the arcs in the interferogram due to topographic errors and deformation rates have the following expression:

$$
\begin{array}{r}
\Delta \boldsymbol{\Phi}_{\text {topo+defo }}^{i}=\left(\underset{G \times(P-1)}{\mathbf{C}} \otimes \underset{1 \times 2}{\mathbf{B}^{i}}\right) \underset{(2 \times(P-1) \times 1)}{\mathbf{P}_{\text {par }}}, \\
i=1, \ldots, M .
\end{array}
$$

The phase differences at the arcs in all interferograms due to topographic errors and deformation rates can then be expressed as

$$
\underset{(M \times G) \times 1}{\Delta \boldsymbol{\Phi}_{\text {topo }+ \text { defo }}}=\underset{(M \times G) \times(2 \times(P-1))}{\mathbf{D}_{\text {par }}} \underset{(2 \times(P-1) \times 1)}{\mathbf{P}_{\text {par }}}
$$

with

$$
\mathbf{D}_{\text {par }}=\left[\begin{array}{llll}
\mathbf{C} \otimes \mathbf{B}^{1} & \mathbf{C} \otimes \mathbf{B}^{2} & \ldots & \mathbf{C} \otimes \mathbf{B}^{M}
\end{array}\right]^{\mathrm{T}} .
$$

\section{Observation Equation and Initial Solution}

The final observation equation reflecting the relationship between the phase differences at the arcs and the unknowns (i.e., orbital error polynomial coefficients, topographic errors, and deformation rates) can be expressed as

$$
\underset{(M \times G) \times 1}{\Delta \boldsymbol{\Phi}}=\mathbf{D P}+\underset{(M \times G) \times 1}{\mathbf{W}}
$$

with

$$
\begin{aligned}
& \mathbf{D}=\left[\begin{array}{ll}
\mathbf{D}_{\text {orb }} & \mathbf{D}_{\text {par }}
\end{array}\right]^{\mathrm{T}} \\
& \mathbf{P}=\left[\begin{array}{ll}
\mathbf{P}_{\text {orb }} & \mathbf{P}_{\text {par }}
\end{array}\right]^{\mathrm{T}}
\end{aligned}
$$

where $\mathbf{W}$ is a vector that contains all the unmodeled phase at the arcs due to, e.g., spatially uncorrelated atmospheric delays and the decorrelation noise. It should be noted that (17) is a large sparse linear system and the direct solution of unknowns relies on matrix factorizations. Sparse factorization is typically used and can be performed using CHOLMOD package [22] that appears in Matlab as a built-in backslash operator. As an alternative, (17) can also be resolved by an iterative method based on the bidiagonalization procedure of Golub and Kahan [23], [24] that appears in Matlab as a built-in LSQR function.

\section{E. Phase Ambiguity Detection and Final Solution}

Since the observations in (17) are wrapped phase, a number of observations might contain phase ambiguities. The initial solution carried out without considering the phase ambiguities should be updated by detecting and removing the observations with modulo $-2 \pi$ [8], [9]. By analyzing the phase residuals from the initial solution, we notice that phase ambiguities can result in abnormally large least squares residuals (Fig. 2). Therefore, the residuals of the initial solution can be used to identify observations with phase ambiguities. For simplicity, we adopt a strategy proposed in [8] that examines the largest residual in each arc, and if the residual is larger than a given threshold, all observations at the arc will be removed from the solution. The advantage of the method is that a strict outlier diagnosis is not required since we do not need to exactly know which observations have phase ambiguities. Instead, we are 
(a)

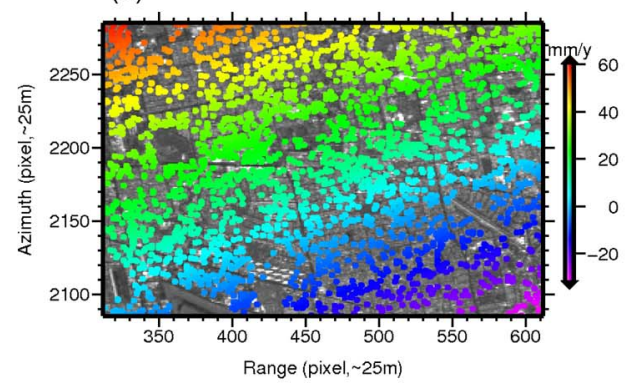

(d)

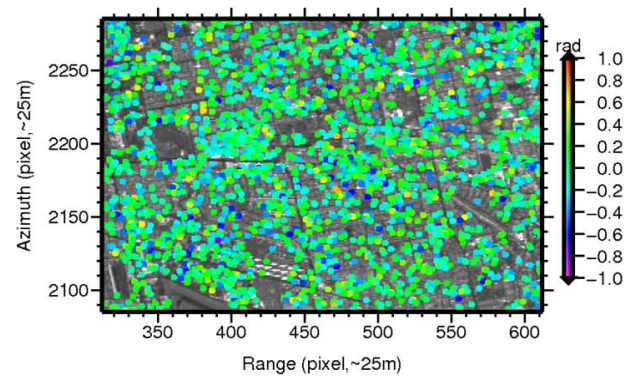

(b)

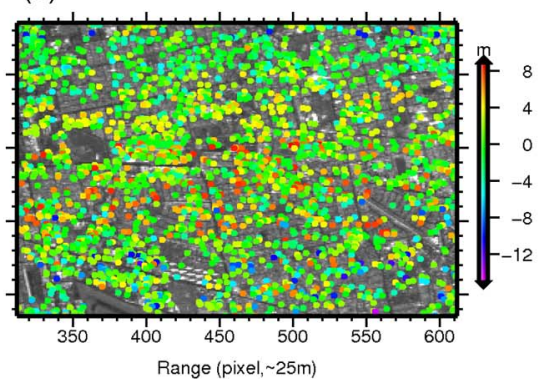

(e)

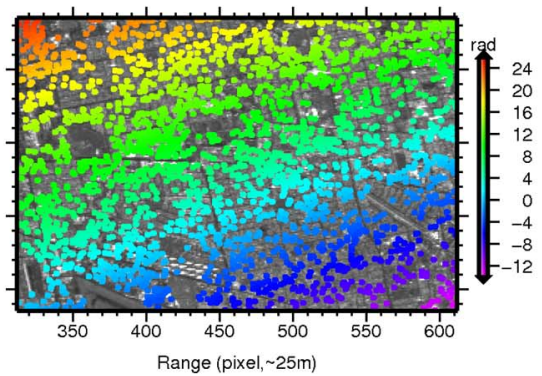

(c)

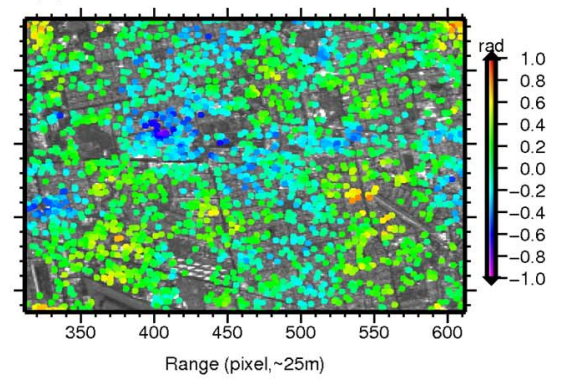

(f)

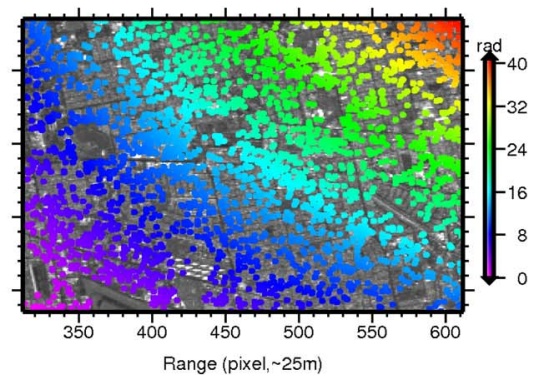

Fig. 3. Examples of simulated signals. (a) Deformation rates. (b) Topographic errors. (c) Atmospheric delays. (d) Noise. (e and f) Orbital errors in two images.

concerned with whether the phase differences of the arc have any ambiguity. Accordingly, a phase ambiguity detector can be constructed as follows:

$$
\operatorname{Max}\left(\left|\mathbf{v}_{g}\right|\right)>\Gamma, \quad g=1, \ldots, G
$$

where $\operatorname{Max}(\cdot)$ denotes the maximum value of the vector, $\mathbf{v}_{g}$ is the vector of phase residuals of the $g$ th arc, and $\Gamma$ is the threshold that is set according to the variance [8] or the histogram of phase residuals. When the condition in (19) is met, the arc will be removed from the final solution.

After the arcs with phase ambiguities are detected and removed, the sparse-matrix least squares is performed again on the remaining arcs to get the unknowns. Finally, we can obtain the solution of the observation model, which includes relative orbital phase errors at SAR acquisition dates with respect to the reference image, DEM errors, and deformation rates at all coherent points. The Matlab routines for the proposed model can be found in the demo codes [25].

\section{Algorithm Testing With Synthetic Data Sets}

To assess the performance of the proposed model, a test with synthetic data was conducted first. The use of synthetic data allows a quantitative analysis. In this test, we simulated five types of signals at 2335 points in a small area of roughly $5 \mathrm{~km} \times 7.5 \mathrm{~km}$ in the Los Angeles Basin, California. The signals include linear deformation rates, topographic errors, atmospheric signals, orbit errors, and random noise. To test the proposed model for extreme cases, the simulated deformation rates [Fig. 3(a)] share exactly the same spatial pattern as the orbital errors in the first SLC image. The simulated topographic errors [Fig. 3(b)] have a uniform distribution between $9 \mathrm{~m}$ and $-15 \mathrm{~m}$. Atmospheric signal is simulated for each image using fractal surfaces with the fractal dimensions being 2.67 [11]. The maximum variation of the simulated atmospheric signal over the area is about $2 \mathrm{rad}$. An example of the atmospheric signal is shown in Fig. 3(c). Since the phase screen caused by the errors in the SAR sensor state vectors is usually spatially smooth in an interferogram, we simulate the orbit errors by using a set of 2-D second-order polynomials. The simulated orbital error for each image varies from $\sim-14 \mathrm{rad}$ to $\sim 40 \mathrm{rad}$ in an area of $5 \mathrm{~km} \times$ $7.5 \mathrm{~km}$, which is much more serious than the normal case. The random noise in all images is randomly set to a mean of $15^{\circ}$ with a standard deviation of $5^{\circ}$. As an example, the phase noise for an image is shown in Fig. 3(d). Fig. 3(e) and (f) shows the typical orbital errors associated with SLC images, which are not the real phases and simply used for generation of orbital phases in interferograms. More details about the simulation can be found in [31]. In the test, a total number of 26 interferograms (Fig. 4) with baselines shorter than $300 \mathrm{~m}$ and 2 years are generated from 19 SLC images containing different types of signals mentioned. A dense point network is first constructed [Fig. 5(a)] with the method of local triangulation [8], and initial parameter estimation is then carried out. By using a threshold value of $2 \mathrm{rad}$ according to the histogram of the residuals [Fig. 5(b)], the arcs potentially with phase ambiguities are removed. This process results in a new network [Fig. 5(c)] for final parameter estimation. The estimated deformation rates are shown in Fig. 6(a). The difference between the estimated deformation rates and the true values is shown in Fig. 6(b) and (c), which has a mean value of $0.1 \mathrm{~mm} / \mathrm{y}$ and a standard deviation of $0.44 \mathrm{~mm} / \mathrm{y}$. Compared with the true orbital errors at each SLC images, the estimated orbital polynomials have errors with a mean of $0.01 \mathrm{rad}$ and a standard deviation of $0.2 \mathrm{rad}$. It is clear from the results that the proposed model is able to isolate effectively the deformation signal from the orbit errors even though they share exactly the same spatial pattern. However, it is worth noting that the pattern of the difference is quite similar with that of atmospheric artifacts, indicating that 


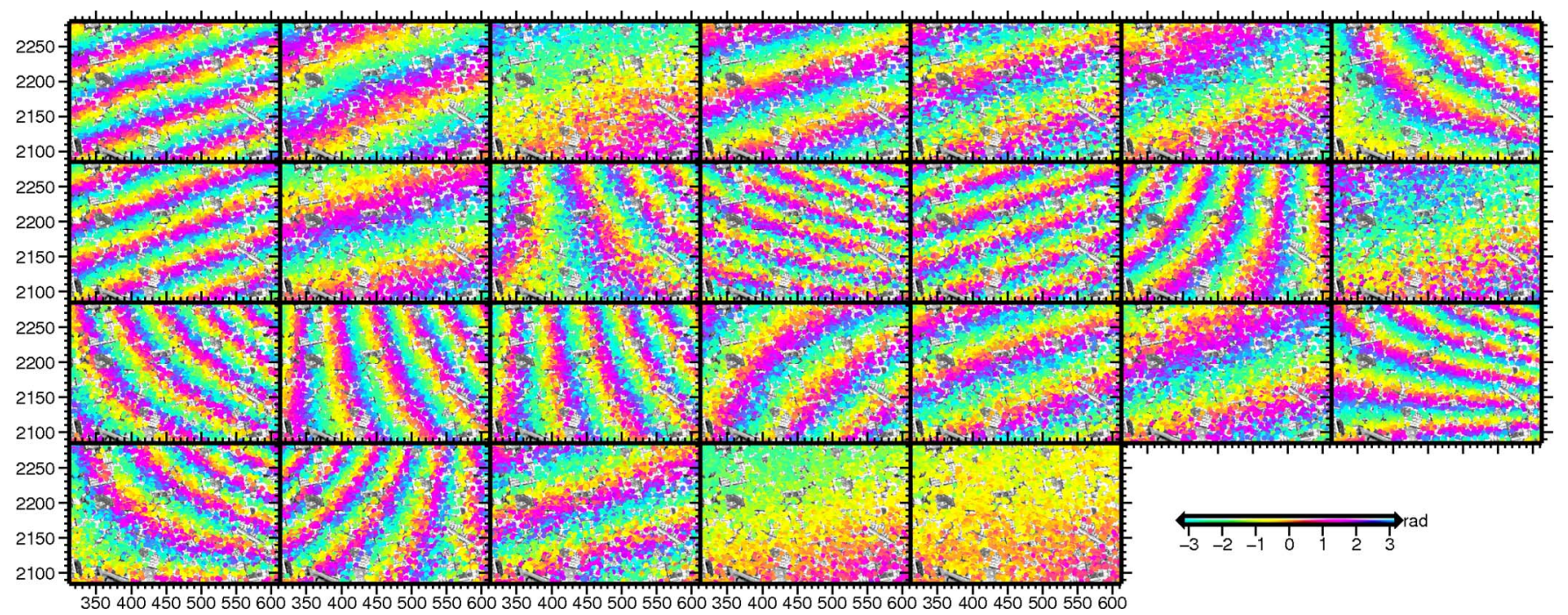

Fig. 4. Simulated interferograms used as observations for the proposed model.

(a)

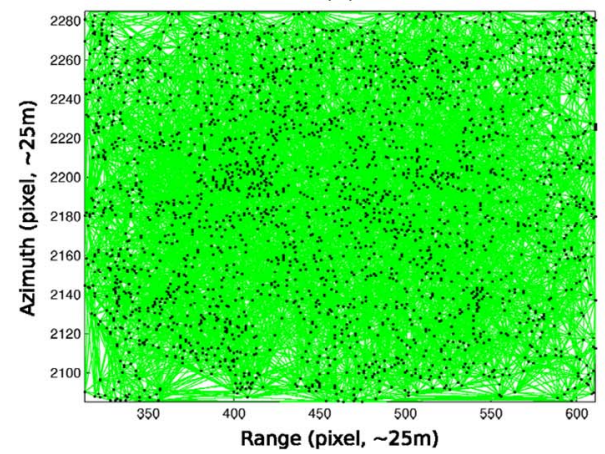

(b)

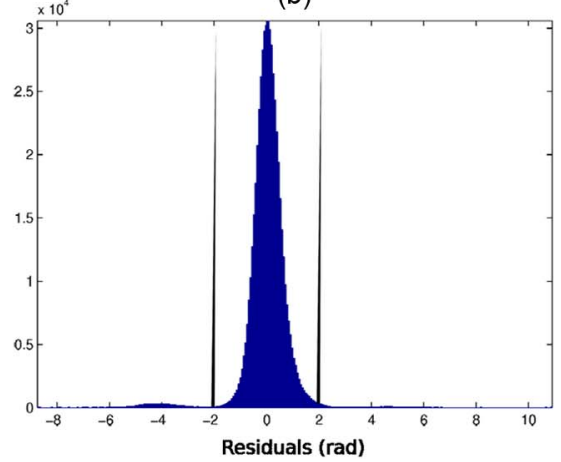

(c)

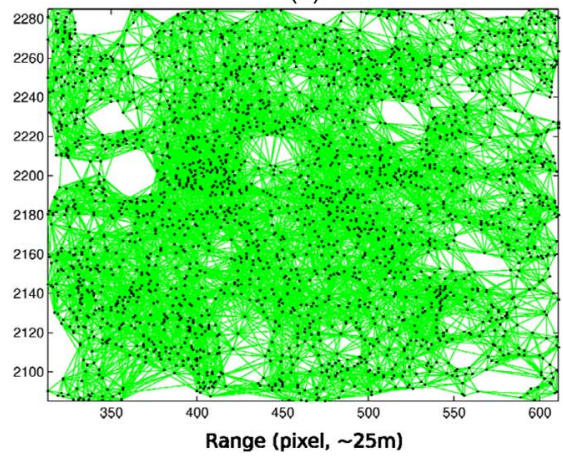

Fig. 5. Phase ambiguity detection. (a) Original network constructed by local Delauany triangulation. (b) Histogram of residuals after the initial parameter estimation. (c) Network updated after removing arcs whose residuals fall within the tails of the histogram determined with a threshold of \pm 2 rad.

(a)

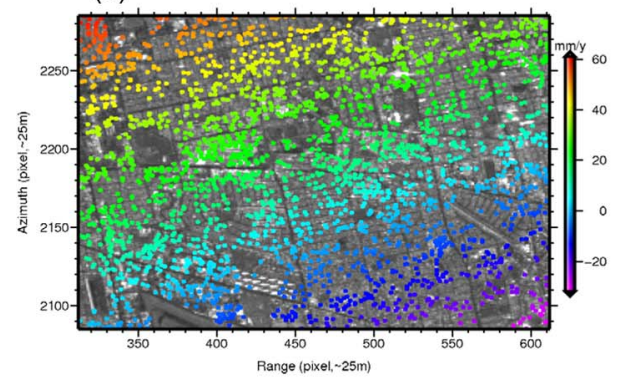

(d)

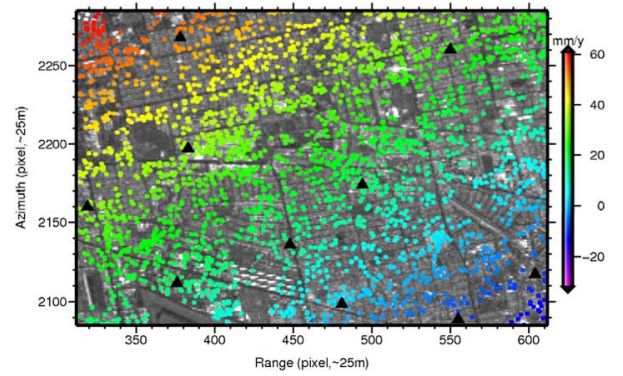

(b)

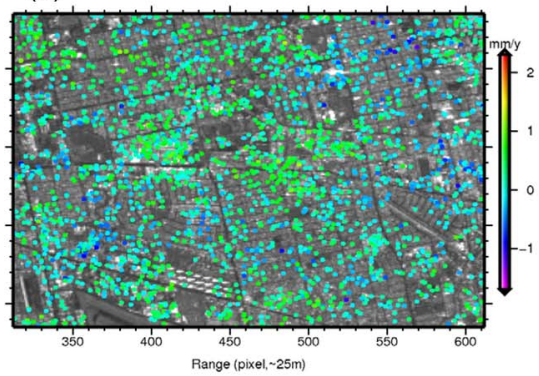

(e)

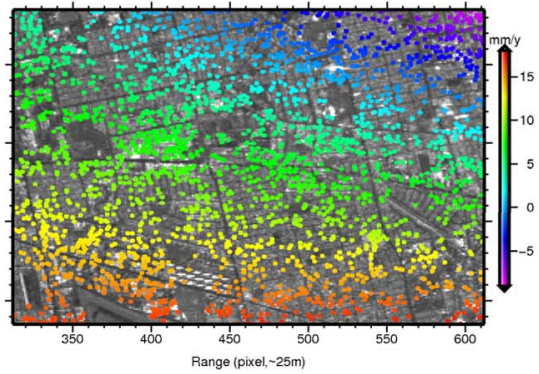

(c)

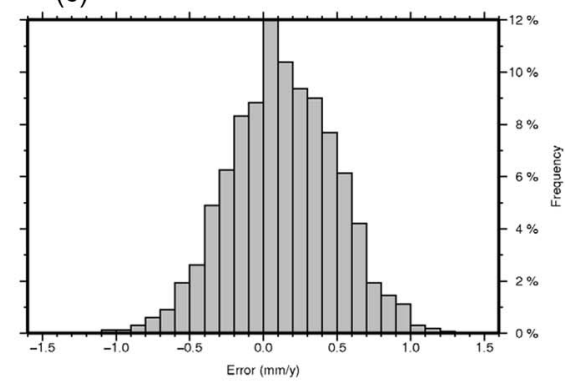

(f)

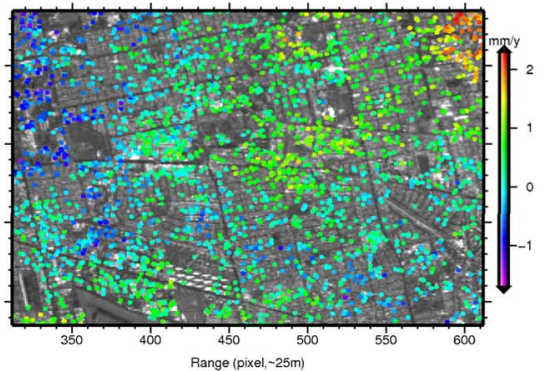

Fig. 6. (a) Estimated deformation rates from interferograms that contain atmospheric artifacts, orbit error, and noise in addition to the deformation signal. (b) Difference between the estimated deformation rates and the true values. (c) Histogram of the difference image in (b). (d) Deformation rates estimated without considering the orbital errors. The black triangles indicate the GCP used for orbital error correction. (e) Orbital polynomial estimated from the GCP. (f) Difference between the estimated deformation rates with GCP-based orbital error correction and the true values. 
TABLE I

ERrors on THE ESTIMATED PARAMETERS UNDER DIFFERENT LEVELS OF ATMOSPHERIC ARTIFACTS

\begin{tabular}{|c|c|c|c|c|}
\hline \multirow{2}{*}{$\begin{array}{c}\text { Range of variation of } \\
\text { atmospheric artifacts with } \\
\text { an area of } 5 \mathrm{~km}-\text { by- } 7.5 \mathrm{~km} \\
(\mathrm{rad})\end{array}$} & \multicolumn{2}{|c|}{$\begin{array}{c}\text { Error in the estimated } \\
\text { deformation rates }(\mathrm{mm} / \mathrm{y})\end{array}$} & \multicolumn{2}{|c|}{$\begin{array}{l}\text { Error in the estimated } \\
\text { orbital phases (rad) }\end{array}$} \\
\hline & Mean & Std. & Mean & Std. \\
\hline$[-1,1]$ & 0.1 & 0.44 & 0.01 & 0.24 \\
\hline$[-2,2]$ & -0.02 & 0.8 & 0.07 & 0.8 \\
\hline$[-3,3]$ & -0.4 & 1.4 & 0.3 & 1.3 \\
\hline
\end{tabular}

the atmospheric artifacts as a vital error source can degrade the precision of the estimated parameters. Since the atmospheric artifacts are also spatially correlated, although the correlation dimension is usually much smaller than that of the orbital errors, some of the atmospheric errors could be absorbed by the orbital error polynomials. This could degrade the accuracy of the estimated orbital errors, but it should not affect significantly the other estimated parameters, e.g., the deformation rates that are of the primary interest. To better understand the effects of atmospheric artifacts on the parameter estimation, we have simulated three levels of atmospheric artifacts with their ranges of magnitude variation being 2, 4, and $6 \mathrm{rad}$, respectively. The means and standard deviations of the errors in the estimated parameters (i.e., deformation rates and orbit errors) are given in Table I. The results clearly indicate that, although phase differences between neighboring coherent points are commonly used in multitemporal equation systems to reduce the effects of spatially correlated atmospheric artifacts, the estimated parameters can be seriously distorted when the atmosphere term is large. A comparison between the proposed model and the widely used method for orbital error correction based on ground control points (GCPs) is made. The deformation rates [see Fig. 6(d)] are first estimated without considering the orbital errors. Ten GCPs with known deformation rate measurements are then selected over the image. To make the comparison more realistic, Gaussian errors with a standard deviation of $1 \mathrm{~mm} / \mathrm{y}$ are added to the GCP. A polynomial is then estimated from the GCP, and the results are shown in Fig. 6(e). The deformation rate map is finally obtained by subtracting the orbital error polynomial from the results in Fig. 6(d). The errors in the estimated deformation rate map are shown in Fig. 6(f). The mean value and the standard deviation of the errors are 0.26 and $0.59 \mathrm{~mm} / \mathrm{y}$, respectively, which are higher than the corresponding values of the results achieved by the proposed model $(0.1$ and $0.44 \mathrm{~mm} / \mathrm{y})$. Part of the reasons for the results is that the GCP-based method for orbital error correction requires a high-accuracy GCP that is normally unavailable in practice. More limitations of the GCP-based method are discussed in Section IV-B. Other successful tests with different deformation patterns can be found in the demo codes [25].

\section{Algorithm Testing With ReAl DatA}

\section{A. Envisat ASAR Data Over Los Angeles Basin}

The Los Angeles Basin, California, has been used frequently for testing InSAR techniques [19], [26]-[28] as it has mod- erate ground movement and low image decorrelation, and there are dense GPS observations from the Southern California Integrated GPS Network for validating the results. We processed 98 interferograms with baselines less than $150 \mathrm{~m}$ and 500 days using 41 Envisat/Advanced SAR (ASAR) SLC images (Frame 2925, Track 170) acquired between August 7, 2004, and September 25, 2010. Based on the differential phases at a total of 2633791 arcs constructed from 851808 coherent points with a spatial resolution of $\sim 25 \mathrm{~m}$, we jointly solved the linear deformation rate and the orbital errors using the proposed model. Quantitative validation and geophysical interpretation of the estimated deformation result are beyond the scope of this paper. Instead, we have highlighted a comparison between the orbital error refinement from the proposed model and from the GCPs. There are 29 GPS sites within the study area that had observations on the SAR acquisition dates, and there are also coherent points within $100 \mathrm{~m}$ from the GPS sites. The interferograms are unwrapped and compared with the GPS observations to get the phase differences between the GPS observations and their nearest coherent points. The phase differences are then fitted to a second-order polynomial. The polynomial is removed from the corresponding unwrapped interferogram. The corrected phases are wrapped again and shown in Fig. 7 together with the detrended interferograms from the proposed model.

It is seen from the results that both of the methods can remove the orbital fringes, and the results from the two methods agree with each other in general. This demonstrates that the proposed approach works well even without using any GCP. To further evaluate the performance of the proposed the model, similar to the test on the synthetic data set, we have added artificial orbital polynomials to several SLC images over the LA basin and allowed the orbital errors to propagate to the related DInSAR phases. As expected, the added orbital errors can be satisfactorily retrieved based on the joint model. It should be noted that the standard deviation of the daily GPS observations in the radar line-of-sight (LOS) direction is around $4 \mathrm{~mm}$ [29], and therefore, the standard deviation of the differential GPS observations between two observation dates is about $5.6 \mathrm{~mm}$. Due to the measurement error at GPS sites, errors in DInSAR images (atmospheric artifacts and other noise), and the location difference between the GPS site and the nearest coherent point, the orbital phase polynomials retrieved by the GCP-based method over the Los Angeles Basin cannot be considered accurate enough to serve as a reference. Table II shows a comparison between the orbital phases in interferograms shown in Fig. 7 estimated from the joint model and those from GCP-based method in terms of standard deviation and mean of their differences. It is observed that the discrepancy is relatively large in the interferogram of 20050514-20050723, with a mean value of $2.28 \mathrm{rad}$ (standard: $1.59 \mathrm{rad}$ ). We believe that the measurement error at GPS sites and the atmospheric artifact in the DInSAR measurement are the main sources of the discrepancy because the orbital polynomial fitting based on GPS also shows a large inconsistency (rmse: $1.97 \mathrm{rad}$ ) for that interferogram. If we assume that the InSAR-derived LOS measurement for the same time interval has similar accuracy with GPS measurements, it can be concluded that the standard deviation of the orbital phase 


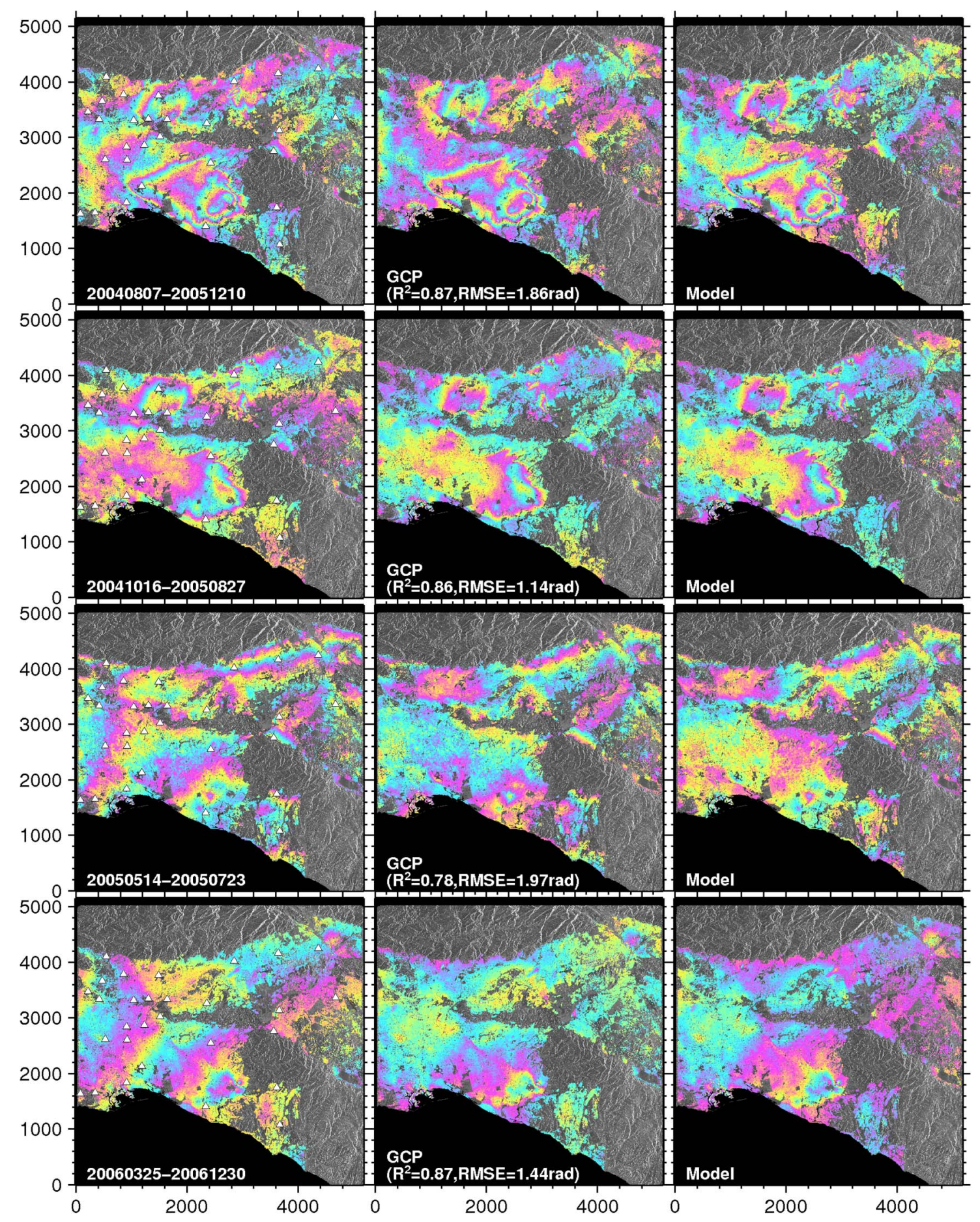

Fig. 7. Comparison between the GCP-based and the proposed approaches for orbit error mitigation. (Left column) Differential interferograms with the locations of CGPS sites marked. (Middle column) Detrended interferograms from the GCP-based approach. (Right column) Detrended interferograms from the proposed approach.

TABLE II

DifFerenCES BetweEn the ORBITAL ERrors Estimated By THE JoINT MOdel AND GCP-BASEd Method

\begin{tabular}{ccc}
\hline \multirow{2}{*}{ Image pairs } & \multicolumn{2}{c}{ Difference between the estimated orbital errors (rad) } \\
\cline { 2 - 3 } & Mean & Std. \\
\hline $20040807-20051210$ & 1.87 & 1.37 \\
$20041016-20050827$ & -0.15 & 0.43 \\
$20050514-20050723$ & 2.28 & 1.59 \\
$20060325-20061230$ & -1.34 & 0.86 \\
\hline
\end{tabular}

estimated from the continuous GPS (CGPS) sites is around $1.8 \mathrm{rad}$. In addition, the DEM errors are also modeled and estimated simultaneously with the orbit error polynomials and the deformation rates in the proposed method. The DEM errors are, however, usually not modeled together with the orbit errors in the GCP-based method. This should also contribute to the discrepancies between the results of the two methods.

Aside from the aforementioned factors that might result in errors of phase differences at GCP, sparse location and poor 


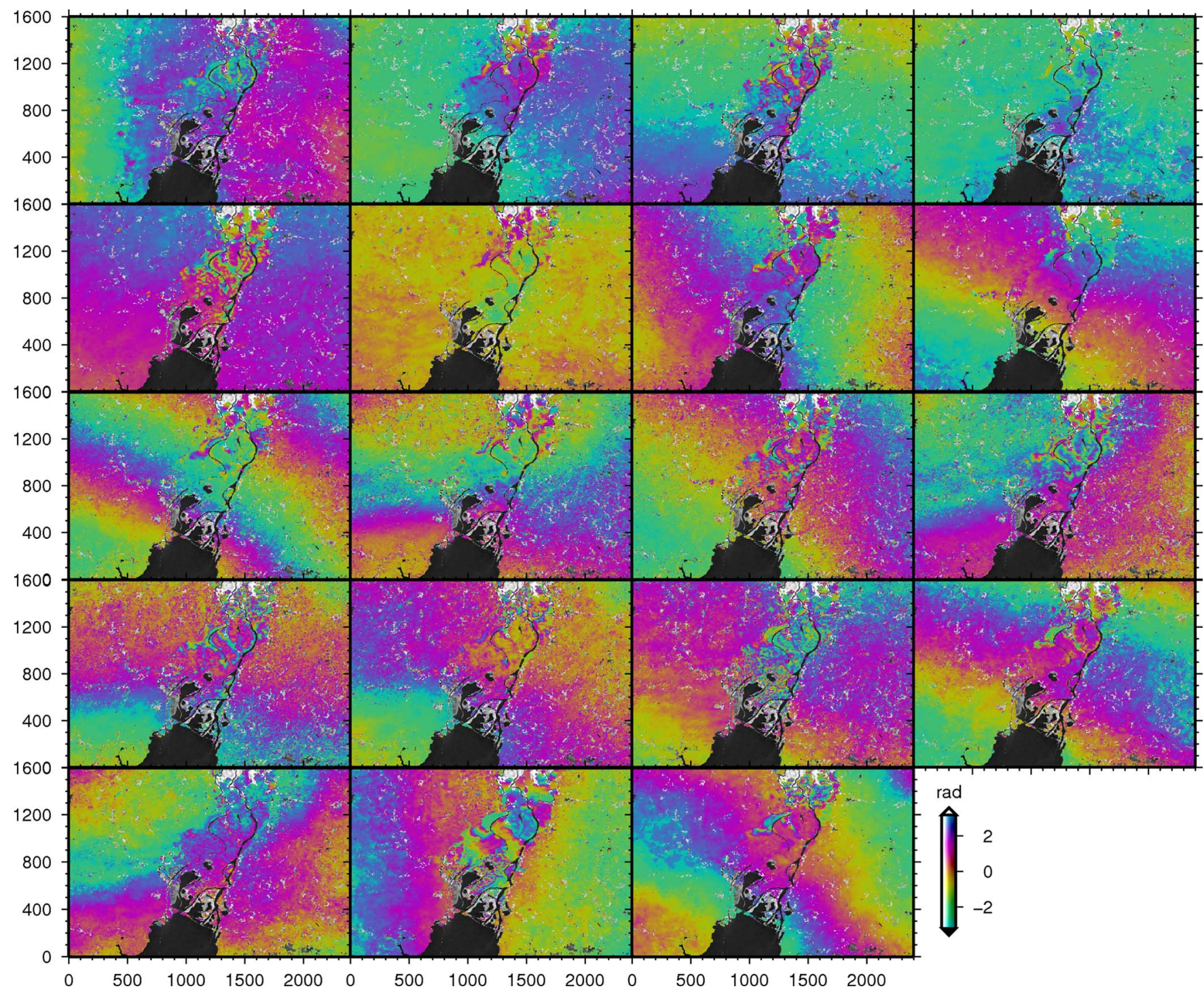

Fig. 8. Wrapped ALOS/PALSAR interferograms over Mobile, Alabama, USA.

spatial distribution of GCP can also adversely affect the polynomial fitting susceptible to edge effects, leading to unrealistic modeling for the orbital error. The GPS density and distribution over Los Angeles are two of the best examples in the real world. Such a luxury of CGPS deployment is not widely available elsewhere, providing a challenge to remove orbital errors in InSAR images based on GCP. On the other hand, our algorithm, which does not rely on any GCP, is an efficient method.

\section{B. ALOS/PALSAR Data Over the City of Mobile}

The City of Mobile is located in the southwestern corner of Alabama, USA. ALOS/PALSAR images are used to map the deformation over the city and its surrounding areas by InSAR techniques. The proposed model is applied to this PALSAR data set since, during the processing, we found that almost all interferograms generated from PALSAR images contain obvious orbital errors. We selected 19 interferograms (Fig. 8) generated from these 12 PALSAR images acquired between June 18, 2007, and August 11, 2010. Differential phases at 1444800 arcs constructed from 422440 coherent points are used as observations, and the resolved LOS linear deformation rates from the proposed model are shown in Fig. 9(a). The results show clear water level change in the wetland area and no notable deformation in the other areas. The map with a color bar ranging from -5 to $5 \mathrm{~mm} / \mathrm{y}$ [Fig. 9(b)] apparently indicates that no observable orbital residuals existed in the estimated deformation. The interferograms after removal of the orbit errors are shown in Fig. 10. It is clear that the orbital fringes have been successfully removed, suggesting that the joint estimation model is adequate.

When applying the proposed model in real cases, it is worth emphasizing that the memory cost might be high. Since the deformation rate, DEM error, and orbital error are simultaneously resolved from the model, a large sparse design matrix is required. For example, for this particular case, the sparse design matrix has a size of $27451200 \times 844933$, and it occupies about 5 GB of memory that nearly doubles that of the matrix without the orbital error parameters. The processing time for this case is about $1188 \mathrm{~s}$ on a desktop PC with an Intel i5 CPU and 16-GB memory, which also about doubles that without orbital error parameters (596 s). 


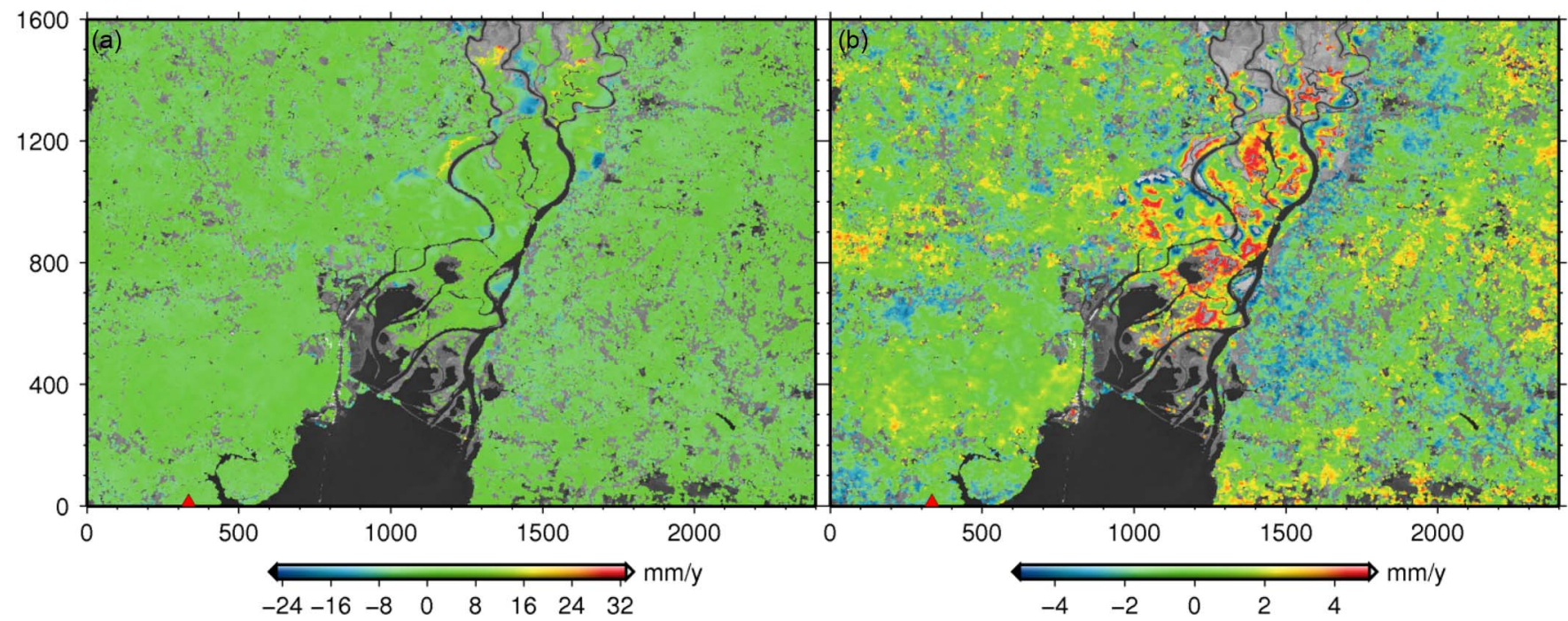

Fig. 9. (a) Estimated linear LOS deformation rate over the City of Mobile. The red triangle is the reference point. (b) Estimated linear LOS deformation rate with a color bar ranging from -5 to $5 \mathrm{~mm} / \mathrm{y}$.

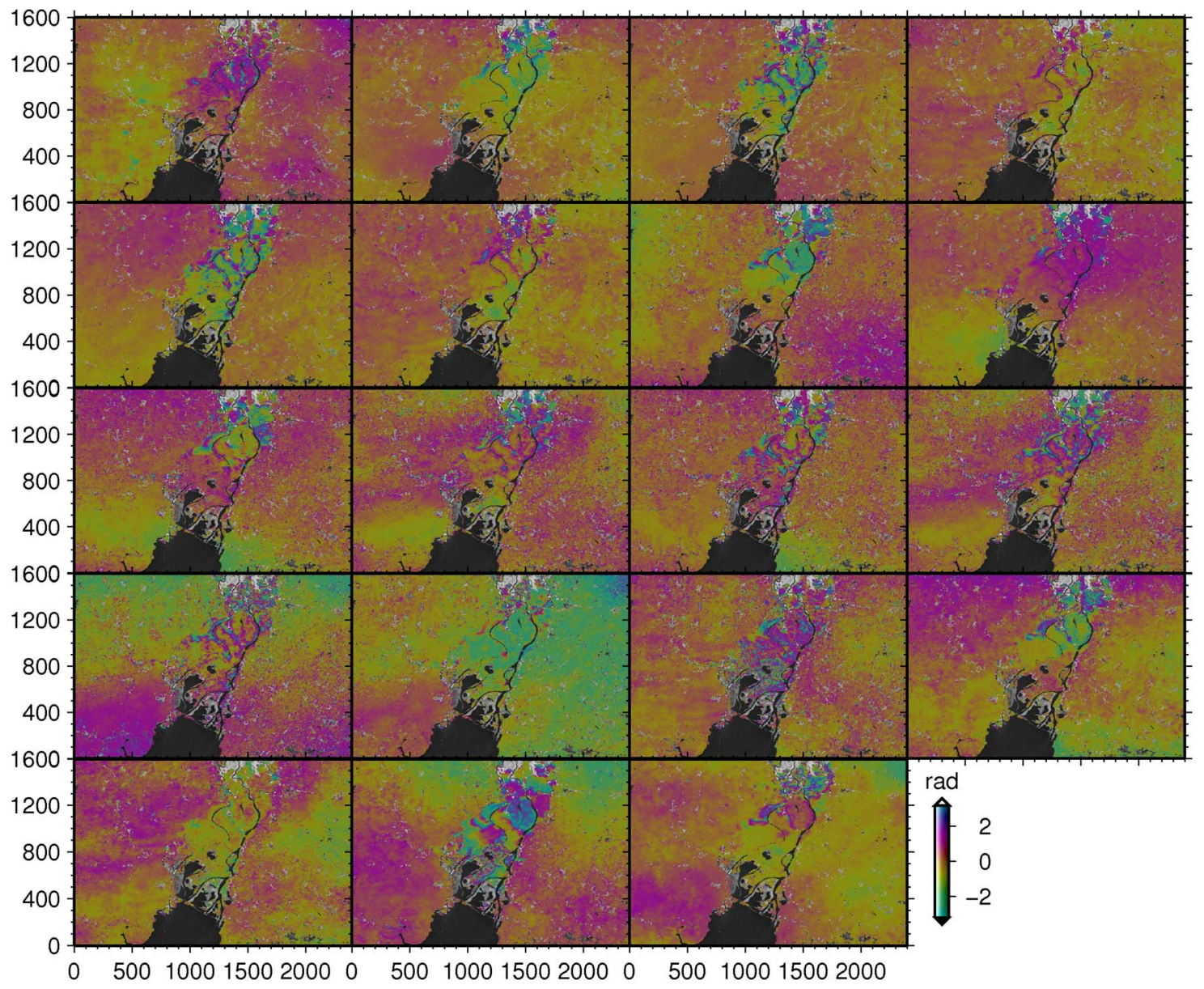

Fig. 10. Interferograms after removing the orbital fringes estimated from the proposed model.

\section{CONClusion AND Discussion}

We have presented a novel least-squares-based MTInSAR model that is able to jointly estimate the deformation signal and orbital errors. A distinctive advantage of the model is that it can isolate the deformation signal from the orbital error without the need of phase unwrapping as well as GCPs. By building up a direct relationship between the differential phases at arcs and the unknown parameters, the model can be solved by sparse least squares, and arcs with phase ambiguities can be detected and removed according to the least squares residuals. In the proposed model, a local triangulation approach [8] is used to ensure that much more observation arcs are included in the network. Such a dense triangulation network is useful for resolving the unknown parameters. 
The quantitative assessment using synthetic images indicates that the joint estimation model can yield results that are within an acceptable range of error. Through experimental results with real SAR data, we have demonstrated that the use of a joint estimation model can yield better separation of deformation signals, thus implying that the proposed model could serve as a promising tool for deformation estimation with SAR data.

It should be noted that the computer memory requirement can become high particularly for a large ground area with dense coherent points. The problem can be resolved by using virtual memory created on the hard disk at the cost of longer processing time when the physical memory is insufficient. Alternatively, the proposed model can be applied to unwrapped phases provided that the phase ambiguities have been reliably determined. In this case, the coherent points can be first subsampled according to their spatial density and the orbital error polynomials, and the deformation rates can be estimated from a subset of sparsely distributed points. After determining the orbital error polynomials, the deformation rates and topographic errors can be resolved for all points. The approach can reduce the size of the design matrix and, hence, the memory requirement significantly.

\section{ACKNOWLEDGMENT}

The Envisat Advanced Synthetic Aperture Radar data over the Los Angeles Basin are copyrighted by the European Space Agency (ESA) and provided by WinSAR and ESA Cat-1 2853. The ALOS/PALSAR data over the City of Mobile are copyrighted by Japan Aerospace Exploration Agency and the Ministry of Economy, Trade, and Industry of Japan and provided by the Alaska Satellite Facility. Several figures were prepared by using Generic Mapping Tools software [33]. The authors would like to thank the associate editor and three anonymous reviewers for their constructive comments and suggestions.

\section{REFERENCES}

[1] A. Ferretti, C. Prati, F. Rocca, and D. di Elettronica, "Nonlinear subsidence rate estimation using permanent scatterers in differential SAR interferometry," IEEE Trans. Geosci. Remote Sens., vol. 38, no. 5, pp. 22022212, Sep. 2000.

[2] B. Kampes and R. Hanssen, "Ambiguity resolution for permanent scatterer interferometry," IEEE Trans. Geosci. Remote Sens., vol. 42, no. 11, pp. 2446-2453, Nov. 2004.

[3] A. Hooper, H. Zebker, P. Segall, and B. Kampes, "A new method for measuring deformation on volcanoes and other natural terrains using InSAR persistent scatterers," Geophys. Res. Lett., vol. 31, no. 23, pp. 611-615, Dec. 2004.

[4] C. Werner, U. Wegmuller, A. Wiesmann, T. Strozzi, G. Sensing, and S. Muri, "Interferometric point target analysis with JERS-1 L-band SAR data," in Proc. IGARSS, Toulouse, France, Jul. 21-25, 2003, pp. 4359-4361.

[5] P. Berardino, G. Fornaro, R. Lanari, and E. Sansosti, "A new algorithm for surface deformation monitoring based on small baseline differential SAR interferograms," IEEE Trans. Geosci. Remote Sens., vol. 40, no. 11, pp. 2375-2383, Nov. 2002.

[6] R. Lanari, O. Mora, M. Manunta, J. Mallorqui, P. Berardino, and E. Sansosti, "A small-baseline approach for investigating deformations on full-resolution differential SAR interferograms," IEEE Trans. Geosci. Remote Sens., vol. 42, no. 7, pp. 1377-1386, Jul. 2004.

[7] P. Blanco, J. J. Mallorquí, S. Duque, and D. Monells, "The coherent pixels technique (CPT): An advanced DInSAR technique for non-linear deformation monitoring," Pure Appl. Geophys., vol. 165, no. 6, pp. 11671193, Jun. 2008.

[8] L. Zhang, X. L. Ding, and Z. Lu, "Modeling PSInSAR time series without phase unwrapping," IEEE Trans. Geosci. Remote Sens., vol. 49, no. 1, pp. 547-556, Jan. 2011
[9] L. Zhang, Z. Lu, X. L. Ding, H. S. Jung, G. C. Feng, and C. W. Lee, "Mapping ground surface deformation using temporarily coherent point SAR interferometry: Application to Los Angeles Basin," Remote Sens. Environ., vol. 117, pp. 429-439, Feb. 2012.

[10] T. R. Lauknes, H. A. Zebker, and Y. Laresen, "InSAR deformation time series using an L1-norm small-baseline approach," IEEE Trans. Geosci. Remote Sens., vol. 49, no. 1, pp. 536-546, Jan. 2011.

[11] R. Hanssen, Radar Interferometry: Data Interpretation and Error Analysis. Dordrecht, The Netherlands: Kluwer, 2001.

[12] Z. Lu, C. Wicks, O. Kwoun, J. Power, and D. Dzurisin, "Surface deformation associated with the March 1996 earthquake swarm at Akutan Island, Alaska, revealed by C-band ERS and L-band JERS radar interferometry," Can. J. Remote Sens., vol. 31, no. 1, pp. 7-20, 2005.

[13] S. Parashar, E. Langham, J. McNally, and S. Ahmed, "RADARSAT mission requirements and concept," Can. J. Remote Sens., vol. 19, no. 4, pp. 280-288, Nov. 1993.

[14] L. C. Morena, K. V. James, and J. Beck, "An introduction to the RADARSAT-2 mission," Can. J. Remote Sens., vol. 30, no. 3, pp. 221234, 2004.

[15] R. Nakamura, S. Nakamura, N. Kudo, and S. Katagiri, "Precise orbit determination for ALOS," in Proc. 20th Int. Symp. Space Flight Dyn., Annapolis, MD, USA, Sep. 24-28, 2007.

[16] A. Pepe, P. Berardino, M. Bonano, L. D. Euillades, R. Lanari, and E. Sansosti, "SBAS-based satellite orbit correction for the generation of DInSAR time-series: Application to RADASAT-1 data," IEEE Trans. Geosci. Remote Sens., vol. 49, no. 12, pp. 5150-5165, Dec. 2011.

[17] T. J. Wright, B. Parsons, P. C. England, and E. J. Fielding, "InSAR observations of low slip rates on the major faults of western Tibet," Science, vol. 305, no. 5681, pp. 236-239, Jul. 2004.

[18] F. Amelung, S. H. Yun, T. R. Walter, P. Segall, and S. W. Kim, "Stress control of deep rift intrusion at Mauna Loa Volcano, Hawaii," Science, vol. 316, no. 5827, pp. 1026-1030, May 2007.

[19] J. Biggs, R. Burgmann, J. T. Freymueller, Z. Lu, B. Parsons, and I. Ryder, "The postseismic response to the 2002 M 7.9 Denali Fault earthquake: Constraints from InSAR 2003-2005," Geophys. J. Int., vol. 176, no. 2 , pp. 353-367, Feb. 2009.

[20] J. Biggs, T. Wright, Z. Lu, and B. Parsons, "Multi-interferogram method for measuring interseismic deformation: Denali fault, Alaska," Geophys. J. Int., vol. 170, no. 3, pp. 1165-1179, Sep. 2007.

[21] H. Bähr and R. F. Hanssen, "Network adjustment of orbit errors in SAR interferometry," in Proc. Fringe, Frascati, Italy, Nov. 30-Dec. 4, 2009, pp. $1-8$.

[22] CHOLMOD. [Online]. Available: http://www.cise.ufl.edu/research/ sparse/cholmod/

[23] G. H. Golub and W. Kahan, "Calculating the singular values and pseudoinverse of a matrix," SIAM J. Numer. Anal., vol. 2, no. 2, pp. 205-224, 1965.

[24] C. C. Paige and M. A. Saunders, "LSQR: An algorithm for sparse linear equations and sparse least squares," ACM Trans. Math. Softw., vol. 8, no. 1, pp. 43-71, Mar. 1982.

[25] Demo Codes of the Proposed Model. [Online]. Available: https://www. dropbox.com/s/1zq5ae9tsqzxwzx/Joint_TCPInSAR_Orbit_Correction_ demo_codes.zip

[26] R. Lanari, P. Lundgren, M. Manzo, and F. Casu, "Satellite radar interferometry time series analysis of surface deformation for Los Angeles, California," Geophys. Res. Lett., vol. 31, no. 23, pp. L23613-1-L23613-5, Dec. 2004.

[27] C. Colesanti, A. Ferretti, C. Prati, and F. Rocca, "Monitoring landslides and tectonic motions with the permanent scatterers technique," Eng. Geol., vol. 68, no. 1/2, pp. 3-14, Feb. 2003.

[28] B. A. Brooks, M. A. Merrifield, J. Foster, C. L. Werner, F. Gomez, M. Bevis, and S. Gill, "Space geodetic determination of spatial variability in relative sea level change, Los Angeles Basin," Geophys. Res. Lett., vol. 34, no. 1, pp. L01611-1-L01611-6, 2007.

[29] F. Casu, M. Manzo, and R. Lanari, "A quantitative assessment of the SBAS algorithm performance for surface deformation retrieval from DInSAR data," Remote Sens. Environ., no. 3/4, pp. 195-210, Jun. 2006.

[30] M. Shirzaei and T. R. Walter, "Estimating the effect of satellite orbital error using wavelet based robust regression applied to InSAR deformation data," IEEE Trans. Geosci. Remote Sens., vol. 49, no. 11, pp. 4600-4605, Nov. 2011.

[31] B. Kampes, Radar interferometry: Persistent Scatterer Technique. Dordrecht, The Netherlands: Springer-Verlag, 2006.

[32] L. Zhang, X. L. Ding, and Z. Lu, "Deformation rate estimation on changing landscapes using temporarily coherent point InSAR," in Proc. Fringe, Frascati, Italy, Sep. 19-23, 2011.

[33] P. Wessel and W. H. F. Smith, "New, improved version of the generic mapping tools released," EOS Trans. AGU, vol. 79, p. 579, 1998. 


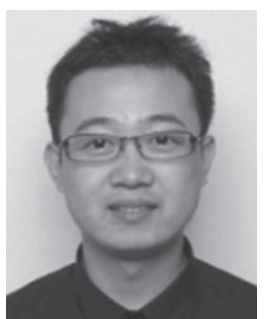

Lei Zhang (S'08-M'11) was born in Yantai, China, in 1981. He received the M.Sc. degree from Tongji University, Shanghai, China, in 2007, with a thesis on fault slip inversion with interferometric synthetic aperture radar (SAR) and GPS data based on a triangular dislocation model and the Ph.D. degree in geodesy and geodynamics from The Hong Kong Polytechnic University, Kowloon, Hong Kong, in 2011.

Since 2012, he has been a Research Assistant Professor with the Department of Land Surveying and Geo-Informatics, The Hong Kong Polytechnic University. His research interests focus on developing advanced processing techniques for SAR images and the application of multitemporal interferometric analysis to the retrieval of geophysical parameters and displacement monitoring, with emphasis on natural hazards.

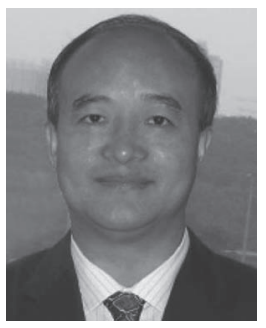

Xiaoli Ding received the B.Eng. degree from Central South University of Metallurgy, Changsha, China, in 1983 and the Ph.D. degree from the University of Sydney, Sydney, Australia, in 1993.

$\mathrm{He}$ is currently the Chair Professor of geomatics and the Head of the Department of Land Surveying and Geo-Informatics, The Hong Kong Polytechnic University, Kowloon, Hong Kong. He lectured at the Northeast University of Technology, Shenyang, China (in 1983-1986), and the Curtin University of Technology, Perth, Australia (in 1992-1996), before joining The Hong Kong Polytechnic University in 1996. His main research interests are in developing technologies for studying ground and structural deformation and geohazards, with a current focus being upon spaceborne geodetic technologies such as GPS and interferometric synthetic aperture radar.

Prof. Ding is the President of Sub-Commission 4.4 of the International Association of Geodesy (IAG) on airborne and spaceborne imaging technologies. $\mathrm{He}$ is also a fellow of the IAG.

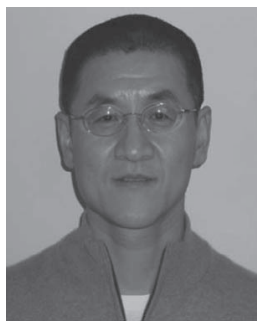

Zhong Lu (S'96-A'97-M'97-SM'07) received the M.S. degree from Peking University, Beijing, China, in 1992 and the Ph.D. degree from the University of Alaska Fairbanks, Fairbanks, AK, USA, in 1996.

$\mathrm{He}$ is currently a Physical Scientist with Cascades Volcano Observatory, U.S. Geological Survey (USGS), Vancouver, WA, USA. He is a Principal Investigator of projects funded by the National Aeronautics and Space Administration (NASA), European Space Agency, Japan Aerospace Exploration Agency, German Aerospace Agency, and USGS on the study of land surface deformation using satellite interferometric synthetic aperture radar (SAR) (InSAR) imagery. He has produced more than 40 leadauthored and 70 coauthored peer-reviewed journal articles and book chapters focused on InSAR techniques and applications. His research interests include technique developments of SAR, InSAR, and persistent scatterer InSAR processing and their applications on natural hazard monitoring and natural resource management.

Dr. Lu was the recipient of an American Society for Photogrammetry and Remote Sensing Award for Best Scientific Paper in Remote Sensing, NASA Group Achievement Award, NASA Certificate of Appreciation, Raytheon Distinguished Level Award for Excellence in Technology, Science Applications International Corporation Technical Fellow, and Jerald Cook Memorial Award. $\mathrm{He}$ is a Committee Member of the International User Team for Radarsat-C SAR Constellations, the GeoEarthscope InSAR User Working Group, the Alaska Satellite Facility UserWorking Group of NASA, and NASA Deformation, Ecosystem Structure, and Dynamics of Ice-Radar Science Definition Team.

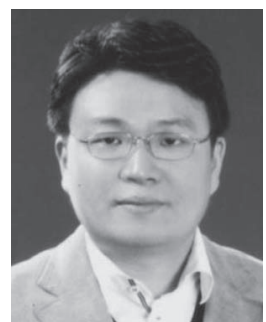

Hyung-Sup Jung (M'09) received the M.S. and $\mathrm{Ph} . \mathrm{D}$. degrees in geophysics and remote sensing from Yonsei University, Seoul, Korea, in 1998 and 2007 , respectively.

$\mathrm{He}$ is currently an Assistant Professor with the Department of Geoinfomatics, The University of Seoul, Seoul. His primary research interests include developments of synthetic aperture radar (SAR), interferometric SAR (InSAR), multiple-aperture InSAR (MAI), and small baseline subset (satellitebased augmentation system) InSAR processors, and algorithms related to 3-D deformation mapping by combining MAI and InSAR and 2-D surface velocity estimation by combining MAI and along-track interferometry.

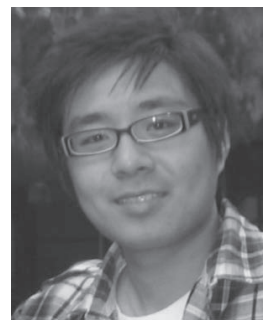

Jun Hu was born in Jiujiang, China, in 1983. He received the M.Eng. degree in geodesy and surveying engineering from Central South University, Changsha, China, in 2008, where he is currently working toward the Ph.D. degree in geodesy and surveying engineering in the Department of Geomatics.

Since September 2012, he has been a Research Associate with the Department of Land Surveying and Geo-Informatics, The Hong Kong Polytechnic University, Kowloon, Hong Kong. His research interests include mapping 3-D surface displacement with interferometric satellite synthetic aperture radar technique and its applications in geophysical fields.

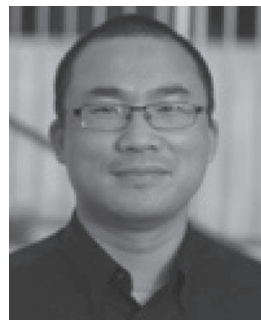

Guangcai Feng received the M.S. degree in geodesy from Central South University, Changsha, China, in 2006 and the Ph.D. degree in geophysics from The Hong Kong Polytechnic University, Kowloon, Hong Kong, in 2011.

$\mathrm{He}$ is currently a Postdoctoral Fellow with the Crustal Deformation and InSAR Group, King Abdullah University of Science and Technology, Thuwal, Saudi Arabia. His research interests are using geodetic techniques (e.g., interferometric synthetic aperture radar, GPS, and leveling) to study the geophysical processes that cause deformation of the crust, e.g., earthquake cycle, earth fissuring, urban subsidence, and landslides, and derive ionospheric perturbations. 